\title{
RELACIÓN ENTRE GRADO CLIINICO DE RINITIS Y FUNCIÓN PULMONAR EN NIÑOS Y ADOLESCENTES SIN ASMA.
}

\section{RELATIONSHIP BETWEEN RHINITIS SEVERITY AND LUNG FUNCTION IN CHILDREN AND ADOLESCENTS WITHOUT ASTHMA. \\ RELAÇÃO ENTRE GRAU CLÍNICO DE RINITE E FUNÇÃO PULMONAR EM CRIANÇAS E ADOLESCENTES EM ASMA.}

\author{
Ricardo José Saranz ${ }^{1,3}$, María Fernanda Agresta ${ }^{2,4}$, Natalia Andrea Lozano ${ }^{1,5}$, Graciela Alegre ${ }^{1,6}$, Laura Verónica \\ Sasia $^{1,7}$, Luciano laniero ${ }^{2,8}$, Yanina Viviana Berardi ${ }^{1,9}$, Alejandro Lozano ${ }^{1,10}$.
}

1-Médicos de Planta, Servicio de Alergia e Inmunología, Clínica Universitaria Reina Fabiola, Universidad Católica de Córdoba, Córdoba, Argentina. Cátedra de Inmunología, Facultad de Ciencias de la Salud, Universidad Católica de Córdoba.

2-Cátedra de Inmunología, Facultad de Ciencias de la Salud, Universidad Católica de Córdoba.

3-ORCID: https://orcid.org/0000-0002-9716-1825

Email de contacto: ricardosaranz@gmail.com;

rsaranz@arnet.com.ar

4-ORCID ID: http://orcid.org/0000-0003-1157-1987

5-ORCID ID: https://orcid.org/0000-0002-1235-2105

6-ORCID ID: https://orcid.org/0000-0001-8391-186X

7-ORCID ID: https://orcid.org/0000-0002-2478-2532

8-ORCID ID: https://orcid.org/0000-0001-7274-3223

9-ORCID ID: https://orcid.org/0000-0002-2433-6460

10-ORCID ID: https://orcid.org/0000-0002-1475-0676

\section{Conceptos clave:}

Que se sabe sobre el tema: Se ha establecido que la rinitis se asocia con alteraciones de la función pulmonar sin expresión clínica.

Que aporta este estudio: La afectación de la función pulmonar se relaciona con los distintos grados clínicos de rinitis.

Recibido: 2019-03-20 Aceptado: 2019-05-12

DOI: http://dx.doi.org/10.31053/1853.0605.v76.n3.23761

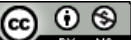

(C) Universidad Nacional de Córdoba

\section{Resumen:}

Introducción: Las vías aéreas superior e inferior se comportan como una unidad. Se han observado alteraciones espirométricas subclínicas en pacientes con rinitis, sin asma, que podrían ser proporcionales a la magnitud de la rinitis. Objetivos: Investigar las posibles alteraciones de la función pulmonar y reversibilidad al broncodilatador en niños y adolescentes con rinitis alérgica (RA) y no alérgica (RNA), sin asma, según el grado clínico de rinitis establecido por ARIA (Rinitis Alérgica y su Impacto en Asma). Población y métodos: Estudio transversal analítico. Se incluyeron pacientes entre 5 y 18 años con RA y RNA, sin asma. Se analizó la existencia de anormalidades en la función pulmonar (curva flujo-volumen) y la respuesta broncodilatadora en relación al grado clínico de rinitis por ARIA ajustando un modelo logístico. Resultados: Se estudiaron 193 pacientes; $42(21,7 \%)$ tuvieron al menos un parámetro espirométrico alterado. Los pacientes con rinitis persistente moderada-grave presentaron mayor afectación de la función pulmonar respecto a otros grados de rinitis $(p=0,009)$. El defecto se asoció a la frecuencia $(p=0,03)$ y a la gravedad de la rinitis $(p=0,04)$ pero no con la atopia $(p=0,28)$. La respuesta broncodilatadora positiva fue más frecuente en los grados de rinitis moderada-grave que en los leves $(p=0,04)$. Conclusiones: La alteración de la función pulmonar fue más prevalente en la rinitis persistente moderada-grave y se asoció a la frecuencia y la gravedad de la rinitis pero fue independiente de la condición de atopia. La reversibilidad al broncodilatador se observó en pacientes con rinitis intermitente y persistente moderada-grave.

Palabras clave: rinitis; clasificación; espirometría.

\section{Abstract:}

Background: The link between upper and lower airways is recognized clinically as a "unique airway". Subclinical spirometric abnormalities have been observed in patients with rhinitis without asthma, which could be proportional to rhinitis severity. Objectives: To investigate possible subclinical alterations in lung function and bronchodilator reversibility in children and adolescents with allergic (AR) and non-allergic rhinitis (NAR) without asthma, according to the clinical grade of rhinitis classified by ARIA (Allergic Rhinitis and Its Impact on Asthma).

Methods: In a cross-sectional analytical study, we included patients aged 5 to 18 years with symptoms of AR and NAR without asthma. Spirometry was performed by flowvolume curve and we analyzed the abnormalities in respiratory function and bronchodilator response in relation to clinical grade of rhinitis by ARIA using an adjusted logistic model. Results: We studied 193 patients; 42 (21.7\%) had some spirometric abnormalities. Patients with moderate-severe persistent rhinitis had greater impairment of lung function compared to the other grades of rhinitis $(p=0.009)$. This defect was associated with both frequency $(p=0.03)$ and severity of rhinitis $(p=0.04)$ but not with atopic status $(p=0.28)$. A positive bronchodilator response was more frequent in grades moderate-severe of rhinitis than in mild forms $(p=0.04)$. Conclusion: Abnormalities of lung function was more prevalent in moderate-severe persistent rhinitis and was associated with the frequency and severity of rhinitis but not to atopic status. The bronchodilator reversibility was observed in patients with intermittent and persistent moderate-severe rhinitis.

Keywords: rhinitis; classification; spirometry.

\section{Resumo}

Introdução: As vias aéreas superiores e inferiores se comportam como uma unidade. Foram observadas alterações espirométricas subclínicas em pacientes com rinite, sem asma, que pode ser proporcional à magnitude da rinite. Objetivos: Investigar as possíveis alterações na função pulmonar e reversibilidade ao broncodilatador em crianças e adolescentes com rinite alérgica (AR) e não alérgica (RNA), sem asma, de acordó com o grau clínico de rinite estabelecido pela ARIA (Rinite Alérgica e seu Impacto sobre Asma). População e métodos: Estudo analítico de corte transversal, que incluiu pacientes entre 5 e 18 anos com AR e RNA, sem asma. Analisou-se a existência de anormalidades na função pulmonar (curva de fluxo-volume) e a resposta broncodilatadora em relação ao grau clínico de rinite pela ARIA ajustando um modelo logístico. Resultados: 193 pacientes foram estudados; $42(21,7 \%)$ apresentavam pelo menos um parâmetro espirométrico alterado. Os pacientes com rinite persistente moderada-grave apresentaram maior comprometimento da função pulmonar em relação aos outros graus de rinite $(p=0,009)$. O defeito foi relacionado com a frequência $(p=0,03)$ e a gravidade da rinite $(p=0,04)$, mas não com atopia $(p=0,28)$. A resposta positiva ao broncodilatador foi mais frequente nos graus de rinite moderada-grave do que nos leves $(p=0,04)$. Conclusões: $A$ alteração na função pulmonar foi mais prevalente em rinite persistente moderada a grave e foi associado com a frequência e a gravidade da rinite, mas foi independente da condição de atopia. A reversibilidade ao broncodilatador foi observada em pacientes com rinite moderada a grave intermitente e persistente.

Palavras-chave: rinite; classificação; espirometria. 


\section{Introducción}

La rinitis es una enfermedad inflamatoria crónica de vía aérea superior que se caracteriza por la presencia de rinorrea, prurito, bloqueo y estornudos que afecta entre el $20 \%$ y el $40 \%$ de la población infantil, y que, por su etiología, puede ser alérgica (RA) o no alérgica $(\mathrm{RNA})^{1,2}$.

La rinitis adquiere relevancia no sólo por su elevada prevalencia sino por el compromiso de la calidad de vida de quienes la padecen, por lo que la guía ARIA (Allergic Rhinitis and its Impact on Asthma) ${ }^{1}$, con fines clínicos, ha propuesto una clasificación teniendo en cuenta la frecuencia y gravedad de síntomas en cuatro grados clínicos: intermitente leve y moderada-grave y persistente leve y moderada-grave.

Múltiples evidencias sustentan una estrecha relación entre la vía aérea superior e inferior ${ }^{3-7}$. Los fenotipos alérgico y no alérgico de rinitis presentan algunas similitudes antes no consideradas. Ambas tienen sintomatología parecida, se asocian a las mismas comorbilidades $^{8} \mathrm{y}$, además, el riesgo de asma está aumentado en niños y adultos con rinitis alérgica y no alérgica con respecto a la población general ${ }^{3,9}$.

La existencia de rinitis puede contribuir a un menor control del asma $^{10-12}$ y existe una concordancia entre la gravedad de la rinitis $y$ la magnitud de los síntomas de asma en pacientes en quienes coexisten ambas enfermedades ${ }^{13}$.

Se han observado alteraciones subclínicas en la función pulmonar de pacientes con rinitis alérgica y no alérgica que no manifiestan asma $^{14-17}$, con reactividad incrementada en la respuesta broncodilatadora $^{18}$, hallazgos que podrían ser la expresión de una enfermedad común que afecta a toda la vía respiratoria.

En un estudio previo ${ }^{19}$ estimamos la presencia de anormalidades espirométricas en niños y adolescentes con RA y RNA en relación a la frecuencia (intermitente vs persistente) y gravedad de síntomas (leve vs moderada vs grave), clasificadas según una propuesta modificada de la guía ARIA (ARIA m) ${ }^{20}$. Teniendo en cuenta el correlato clínico funcional mencionado entre rinitis y asma estimamos probable que la afectación subclínica de la función pulmonar sea directamente proporcional a la magnitud de la rinitis considerando los cuatro grados clínicos de rinitis según ARIA original: quienes conjuntamente presentan síntomas más frecuentes y mayor afectación de la calidad de vida, podrían tener mayor limitación en sus parámetros funcionales medidos por curva flujovolumen, con potenciales implicancias diagnósticas, pronósticas y terapéuticas. Por lo tanto, consideramos relevante investigar las posibles alteraciones subclínicas de la función pulmonar y su potencial reversibilidad al broncodilatador en niños y adolescentes con rinitis alérgica y no alérgica, sin asma, en relación al grado clínico de rinitis clasificado por ARIA original.

\section{Población y Métodos}

Diseño del estudio: Estudio transversal, observacional, analítico.

\section{Criterios de inclusión y exclusión}

Se incluyeron niños y adolescentes de ambos sexos, entre 5 y 18 años asistidos en el Servicio de Alergia e Inmunología de la Clínica Universitaria Reina Fabiola, Universidad Católica de Córdoba, Argentina con diagnóstico de rinitis según la presencia de dos o más síntomas nasales característicos (rinorrea, bloqueo, prurito y/o estornudos). Se incluyeron en forma sucesiva, pacientes con rinitis alérgica y no alérgica, de acuerdo a la existencia o no de sensibilidad a aeroalergenos determinada por pruebas cutáneas de hipersensibilidad inmediata y se dividieron en cuatro grupos clínicos establecidos en la guía ARIA ${ }^{1}$.

Se excluyeron los pacientes con las siguientes condiciones:

a. Antecedente de asma o de síntomas equivalentes por historia clínica.

b. Historia de infección respiratoria grave (ej. bronquiolitis).

c. Infección aguda o crónica de vías aéreas superiores. d. Alteraciones nasales anatómicas, hipertrofia adenoidea y poliposis.

e. Uso previo de inmunoterapia alergeno-específica (subcutánea o sublingual).

f. Medicación con esteroides tópicos o sistémicos, antihistamínicos, antagonistas de leucotrienos y alfa-adrenérgicos (tópicos o sistémicos) en las últimas cuatro semanas.

g. Fumadores activos y/o expuestos al humo de cigarrillo en el ámbito familiar.

\section{Clasificación clínica de la rinitis por grados}

Se estableció de acuerdo a los criterios de ARIA ${ }^{1}$. Según la frecuencia, pacientes que presentaron síntomas menos de cuatro días a la semana por menos de cuatro semanas consecutivas fueron clasificados como rinitis intermitente; la existencia de síntomas por más de cuatro días a la semana por más de cuatro semanas consecutivas fue considerada como rinitis persistente. La gravedad de la rinitis se determinó según el compromiso de la calidad vida (limitaciones en la escolaridad, actividades diarias, sueño y existencia de síntomas molestos), en leve (ningún ítem afectado) y moderada-grave (uno o más de los de los aspectos de calidad de vida comprometidos).

De acuerdo a ello se clasificaron cuatro grupos clínicos de rinitis: Intermitente leve, intermitente moderada-grave, persistente leve y persistente moderada-grave.

\section{Variables estudiadas}

Se consideró edad, sexo, índice de masa corporal (IMC) y duración de la rinitis. Se realizó espirometría por curva flujo-volumen, pruebas cutáneas por puntura con alergenos de lectura inmediata (prick test).

La duración de la rinitis se estableció mediante la diferencia entre edad de inicio de los síntomas y la edad del niño al momento del diagnóstico. El porcentaje de vida afectada fue resultado de la ecuación: [Edad de diagnóstico - edad de inicio/ edad de diagnóstico] x 100 .

Para las pruebas cutáneas con alergenos se utilizó un panel estandarizado con los siguientes alergenos del laboratorio AlergoPharma ${ }^{\circledR}$ (Buenos Aires, Argentina): ácaros (Dermatophagoides pteronyssinus, Dermatophagoides farinae, Blomia tropicales), hongos ambientales (Alternaria sp, Aspergillus sp, Cladosporium, Mucor, Rhizopus, Penicillum), epitelio de perro, epitelio de gato y pólenes de árboles, gramíneas, compuestas y tala, solución salina fenolada (control negativo) e histamina $1 \mathrm{mg} / \mathrm{ml}$ (control positivo).

Las pruebas se efectuaron en la cara anterior del antebrazo, utilizándose una lanceta Pricker (Diater Laboratorios ${ }^{\circledR}$, Buenos Aires, Argentina) con lectura a los quince minutos con regla milimetrada. Se consideró positiva la presencia de una pápula de tres o más milímetros como promedio de la lectura de los diámetros ortogonales en relación al testigo control negativo ${ }^{21}$. La presencia de una o más pruebas cutáneas con alergenos positivas se correspondió con rinitis alérgica; su ausencia fue compatible con el fenotipo de rinitis no alérgica.

La espirometría en modo curva flujo-volumen se realizó con un espirómetro Vitalograf $\AA$ modelo 2120 UK, de acuerdo a las normas internacionales de American Thoracic Society/European Respiratory Society $(A T S / E R S)^{22}$. Se determinaron los parámetros Capacidad Vital Forzada (FVC), Volumen Espiratorio Forzado en el primer segundo de la FVC (FEV1), coeficiente FEV1/FVC y Flujo Espiratorio Forzado entre el $25 \%$ y el $75 \%$ de la Capacidad Vital Forzada (FEF $25-75 \%$ ), calculados directamente por el programa incluido en el aparato utilizado según tabla de valores normales de Knudson ${ }^{23}$. Se consideraron valores anormales inferiores al $80 \%$ para los tres primeros parámetros y al $65 \%$ para FEF $25-75 \%$ respectivamente en relación a los valores predictivos normales ${ }^{22}$. Se valoró la respuesta broncodilatadora luego de 15 minutos de la administración de 200 ug de Salbutamol (Ventolín®, GlaxoSmithKline) con una cámara espaciadora Aerocámara® de $500 \mathrm{ml}$ con válvula unidireccional. Se calificó positiva una mejoría en el FEV1 $\geq$ al $12 \%$ respecto al valor basal pre-broncodilatador mediante el siguiente cálculo: FEV1 post BD - FEV1 previo BD / FEV1 previo $\mathrm{BD} \times 100^{22}$. Se eligió la mejor de tres determinaciones 
basales y tres post broncodilatador, que cumplieran los criterios de aceptabilidad y reproducibilidad según ATS/ERS ${ }^{22}$.

Las pruebas cutáneas con alergenos y la espirometría por curva flujo volumen fueron realizadas por distintos operadores sin conocimiento de las otras variables estudiadas. Para evitar variaciones circadianas, todos los estudios complementarios fueron realizados entre las 09:00 y 12:00 AM.

\section{Aspectos éticos}

El trabajo de investigación fue aprobado por el Comité Institucional de Ética de la Investigación en Salud (CIEIS) de la Clínica Universitaria Reina Fabiola, Facultad de Ciencias de la Salud, Universidad Católica de Córdoba. Se realizó cumpliendo con la normativa de la declaración de Helsinki y Buenas Prácticas Clínicas y de acuerdo con la ley de la Provincia de Córdoba (Argentina) sobre Investigación en seres humanos $n^{\circ}$ 9694/09. Para todas las intervenciones diagnósticas y para el uso de datos se solicitó el consentimiento informado correspondiente y se garantizó su confidencialidad según la ley nacional de protección de datos personales $\mathrm{n}^{\circ} 25326 / 00$.

\section{Análisis estadístico}

Se realizó estadística descriptiva de las variables. Para comparación de variables cuantitativas se utilizó prueba de Kruskal Wallis y para variables categóricas prueba de Chi Cuadrado de Independencia. El análisis de la ocurrencia de alteración en la función pulmonar, cualquiera fuera su origen, de la afectación en cada uno de los parámetros respiratorios y el de la respuesta broncodilatadora positiva en relación al grado de rinitis se realizó ajustando un modelo logístico.

Se consideró un nivel de significación del $5 \%$ para establecer significación estadística. Se utilizó el programa estadístico $\mathrm{R}$ a través de la interfaz de InfoStat ${ }^{24}$.

\section{Resultados}

Se incluyeron 193 niños y adolescentes de ambos sexos, de entre 5 y 18 años (108 varones; $56 \%$ ), cuyas características demográficas y generales se observan en la Tabla 1 . En 42 de 193 pacientes $(21,7 \%)$ se observó al menos un parámetro alterado en la función pulmonar (Tabla 2). Los pacientes con rinitis persistente moderadagrave presentaron mayor afectación de la función pulmonar respecto a los otros grados clínicos de rinitis $(p=0,009)$ (Tabla 3 ).

La existencia de al menos un parámetro de función pulmonar alterado se asoció tanto a la frecuencia $(p=0,03)$ como a la gravedad de los síntomas de rinitis $(p=0,04)$ pero no se relacionó con la condición de atopia $(\mathrm{p}=0,28)$.

La respuesta broncodilatadora positiva se observó más frecuente en pacientes con rinitis intermitente moderada-grave y persistente moderada-grave que en aquéllos con formas leves de rinitis (Tabla 3). La reversibilidad al broncodilatador se asoció a la gravedad $(p=0,04)$ pero no con la frecuencia de rinitis $(p=0,19)$ ni con la sensibilización alérgica $(p=0,63)$.

Tabla $\mathrm{N}^{\circ}$ 1: Características demográficas y generales de niños y adolescentes con rinitis alérgica y no alérgica estudiados según el grado clínico.

\begin{tabular}{lcccc|c}
\hline Variable & $\begin{array}{c}\text { Rinitis intermitente } \\
\text { leve } \\
(\mathbf{n = 2 3})\end{array}$ & $\begin{array}{c}\text { Rinitis intermitente } \\
\text { moderada-grave } \\
(\mathbf{n = 2 7})\end{array}$ & $\begin{array}{c}\text { Rinitis persistente } \\
\text { leve } \\
(\mathbf{n = 4 4})\end{array}$ & $\begin{array}{c}\text { Rinitis persistente } \\
\text { moderada-grave } \\
(\mathbf{n = 9 9 )}\end{array}$ & Valor p \\
\hline Edad (años) & $11,00 \pm 3,85$ & $11,79 \pm 3,00$ & $9,92 \pm 3,05$ & $11,94 \pm 3,32$ & \\
& $(6,08-18,00)$ & $(6,50-18,67)$ & $(5,42-17,00)$ & $(5,08-18,00)$ & 0,006 \\
Peso (kg) & $39,53 \pm 13,04$ & $44,18 \pm 14,17$ & $37,32 \pm 15,60$ & $44,78 \pm 16,51$ & \\
& $(22,00-60,00)$ & $(19,00-75,50)$ & $(17,00-77,00)$ & $(17,30-92,00)$ & 0,03 \\
Talla (metros) & $1,45 \pm 0,17$ & $1,52 \pm 0,16$ & $1,40 \pm 0,17$ & $1,50 \pm 0,18$ & \\
Indice de masa & $(1,20-1,69)$ & $(1,14-1,80)$ & $(1,12-1,73)$ & $(1,13-1,88)$ & 0,01 \\
corporal & $18,38 \pm 3,08$ & $18,67 \pm 2,64$ & $18,19 \pm 3,56$ & $19,10 \pm 3,56$ & \\
Edad inicio síntomas & $(14,48-25,88)$ & $(14,62-24,77)$ & $(11,24-27,58)$ & $(12,64-30,36)$ & 0,42 \\
(años) & $5,72 \pm 3,80$ & $6,07 \pm 4,57$ & $5,77 \pm 3,41$ & $6,26 \pm 3,68$ & \\
Duración de la rinitis & $(1,00-15,00)$ & $(0,50-14,00)$ & $(0,50-14,00)$ & $(0,50-16,00)$ & 0,78 \\
(años) & $5,27 \pm 3,75$ & $5,72 \pm 3,79$ & $4,15 \pm 2,69$ & $5,69 \pm 3,51$ & \\
Porcentaje de vida & $(1,00-15,33)$ & $(0,75-13,00)$ & $(0,25-11,83)$ & $(0,50-17,00)$ & 0,13 \\
afectada & $48,55 \pm 25,08$ & $50,12 \pm 31,40$ & $43,23 \pm 25,63$ & $47,94 \pm 25,24$ & \\
\hline Atópicos & $(7,69-88,46)$ & $(5,08-94,64)$ & $(3,03-94,00)$ & $(5,41-96,00)$ & 0,75 \\
Masculinos & $70 \%$ & $96 \%$ & $62 \%$ & $78 \%$ & 0,01 \\
\hline
\end{tabular}

Los datos se expresan como media \pm DS y (recorrido).

Tabla $\mathbf{N}^{\circ}$ 2: Distribución de la afectación de los parámetros funcionales respiratorios en niños y adolescentes con rinitis estudiados $(n=193)$.

\begin{tabular}{ll}
\hline Parámetro funcional afectado & $\mathbf{n}(\boldsymbol{\%})$ \\
\hline FVC & $17(8,8)$ \\
FEV1 & $20(10,4)$ \\
FEV1/FVC & $20(10,4)$ \\
FEF25-75\% & $18(9,3)$
\end{tabular}

FVC: Capacidad Vital Forzada; FEV1: Flujo espiratorio forzado en el primer segundo de la FVC; FEF25-75\%: Flujo espiratorio medio forzado entre el $25 \%$ y $75 \%$ de la FVC. 
Tabla $\mathrm{N}^{\circ}$ 3: Distribución porcentual de la alteración de la función pulmonar, anormalidades en los distintos parámetros de la curva flujo volumen y respuesta broncodilatadora, según el grado clínico de la rinitis por ARIA.

\begin{tabular}{llllllccc}
\hline Variable & $\begin{array}{c}\text { IL } \\
(\mathbf{n = 2 3})\end{array}$ & $\begin{array}{c}\text { IMG } \\
(\mathbf{n = 2 7})\end{array}$ & $\begin{array}{c}\text { PL } \\
(\mathbf{n}=\mathbf{4 4})\end{array}$ & $\begin{array}{c}\text { PMG } \\
(\mathbf{n = 9 9})\end{array}$ & p-valor & $\begin{array}{c}\text { p-valor } \\
\text { frecuencia } \\
\text { síntomas }\end{array}$ & $\begin{array}{c}\text { p-valor } \\
\text { gravedad } \\
\text { síntomas }\end{array}$ & $\begin{array}{c}\text { p-valor } \\
\text { atópicos }\end{array}$ \\
\hline FP alterada (\%) & $4,1^{\mathrm{b}}$ & $17,5^{\mathrm{b}}$ & $13,1^{\mathrm{b}}$ & $30,3^{\mathrm{a}}$ & 0,009 & 0,03 & 0,04 & 0,28 \\
FVC (\%) & $1,7^{\mathrm{b}}$ & $3,9^{\mathrm{b}}$ & $0,0^{\mathrm{b}}$ & $9,9^{\mathrm{a}}$ & 0,006 & 0,18 & 0,005 & 0,02 \\
FEV1 (\%) & $2,0^{\mathrm{b}}$ & $6,9^{\mathrm{b}}$ & $0,0^{\mathrm{b}}$ & $12,5^{\mathrm{a}}$ & 0,001 & 0,32 & 0,001 & 0,04 \\
FEV1/FVC (\%) & 0,0 & 13,2 & 8,3 & 7,5 & 0,12 & 0,67 & 0,06 & 0,22 \\
FEF 25-75 (\%) & $0,0^{\mathrm{b}}$ & 10,0 & 5,0 & 10,2 & 0,08 & 0,32 & 0,05 & 0,07 \\
Resp.BD $(+)(\%)$ & $0,0^{\mathrm{b}}$ & $9,3^{\mathrm{a}}$ & $4,9^{\mathrm{b}}$ & $10,8^{\mathrm{a}}$ & 0,04 & 0,19 & 0,04 & 0,63 \\
\hline
\end{tabular}

a vs b: p<0,05. El agrupamiento (comparaciones) que surge de las letras debe interpretarse dentro de cada parámetro. IL: Rinitis intermitente leve; IMG: Rinitis intermitente moderada-grave; PL: Rinitis persistente leve; PMG: Rinitis persistente moderada-grave. FP: Función pulmonar; FVC: Capacidad Vital Forzada; FEV1: Flujo espiratorio forzado en el primer segundo de la FVC; FEF25-75\%: Flujo espiratorio forzado entre el 25\% y 75\% de la FVC.; Resp.BD (+): respuesta broncodilatadora positiva.

\section{Discusión}

Este estudio demuestra que algunos pacientes con rinitis sin asma tienen alteraciones funcionales respiratorias detectadas en la curva flujo-volumen que son más prevalentes en aquéllos con mayor gravedad clínica de la rinitis.

Las conexiones fisiológicas y patológicas entre la vía aérea superior e inferior han sido consideradas por diversos autores y reconocidas como una "vía aérea única" en la guía ARIA ${ }^{1}$. Así la nariz y el pulmón son órganos estrechamente relacionados desde el punto de vista epidemiológico, anatómico y funcional, con directas implicancias patológicas, clínicas y terapéuticas ${ }^{5-7}$.

Se ha demostrado que la rinitis en la infancia contribuye a aumentar la morbilidad del asma, generando un mayor uso de fármacos para el control de la enfermedad ${ }^{10}$. Los niños con rinitis alérgica manifiestan un menor control del asma estimado por cuestionario de calidad de vida, respecto a quienes no la presentan ${ }^{11}$; además, su tratamiento tiene un impacto favorable sobre los síntomas concurrentes de asma, la función pulmonar y la hiperreactividad bronquial $^{25}$.

Así como la gravedad de la rinitis influencia el grado de control del asma en pacientes que padecen simultáneamente ambas enfermedades, el impacto sobre la función pulmonar, en niños sin asma, parece ser directamente proporcional a la gravedad de la rinitis. Nuestros resultados son coherentes con la hipótesis de "una vía aérea, una enfermedad" dado que aquéllos niños y adolescentes con rinitis persistente moderada-grave, la forma más grave, tienen una mayor afectación de la función pulmonar que aquéllos pacientes incluidos en los otros grados de rinitis. Esto podría tener una explicación fisiopatológica: la conexión fisiopatogénica relacionada a un proceso sistémico alérgico mediado por eosinófilos, un fenómeno inflamatorio común de alergia local órgano específica y otros mecanismos no alérgicos, podrían contribuir a que la rinitis de cualquier origen tenga un impacto subclínico en la vía áerea intratorácica ${ }^{4,6,26}$. Nuestras observaciones sugieren que, cuanto más grave sea la enfermedad de la vía aérea superior, mayor influencia tendría en la vía aérea inferior, aun en ausencia de asma.

Este estudio indica que tanto el efecto de la frecuencia como el de la gravedad de la rinitis contribuyen al defecto ventilatorio de la función pulmonar. Aquéllos pacientes con mayor compromiso de la calidad de vida, presentaron una afectación significativamente mayor de los parámetros FEV1 y FVC. Llamativamente, el FEF25-75\% fue uno de los parámetros menos comprometidos no observándose diferencias entre los grados clínicos de rinitis. Ello puede ser debido a que consideramos un umbral de anormalidad menor al $65 \%$ del teórico normal lo que incrementó su especificidad en detrimento de la sensibilidad para detectar compromiso funcional.

Además, y en coincidencia con los resultados reportados por otras investigaciones ${ }^{9,14,19}$, el registro de anormalidad en la función pulmonar fue independiente de la existencia de alergia: ambos fenotipos de rinitis, alérgica y no alérgica, presentaron niveles similares de afección. Esto indica que, cuanto más grave sea la enfermedad nasal, mayor impacto tendría en la vía aérea intrapulmonar, pero no relacionado a su etiología.

Otro aspecto relevante es conocer si el defecto ventilatorio pulmonar de estos pacientes es potencialmente reversible ${ }^{18,27}$. En un estudio previo de nuestra autoría, considerando disociadas las condiciones de frecuencia y gravedad de síntomas, la reversibilidad al broncodilatador no fue significativa ${ }^{19}$. Contrariamente, en este estudio, observamos una asociación entre las formas intermitente y persistente moderadas-grave de rinitis y los resultados positivos de la prueba broncodilatadora. Esto podría ayudarnos a definir mejor el grupo de pacientes con riesgo incrementado de padecer asma, ya que al reunir la doble condición de tener la alteración funcional y su reversibilidad, condicionaría un seguimiento clínico más estricto.

De acuerdo con estos hallazgos, es imperativo establecer si el compromiso subclínico de la función pulmonar en pacientes con rinitis constituye un biomarcador pronóstico para el desarrollo de asma. Recientemente Skylogianni et al. ${ }^{28}$ realizó el primer estudio longitudinal en niños con rinitis donde demostró que la existencia de disfunción de la pequeña vía aérea manifiesta por oscilometría forzada (FOT), tiene valor predictivo para el desarrollo de asma, independiente de otras condiciones como eccema, asma materna y sensibilización alérgica. Siendo la rinitis un factor de riesgo para el desarrollo de asma ${ }^{3,9}$, posiblemente dicha evolución dependa de factores genéticos y ambientales comunes o relacionados a las características de la microbiota respiratoria, para una eventual coexistencia futura de rinitis y asma, que podría tener una expresión inicial subclínica en la alteración de la función pulmonar ${ }^{5,29,30}$.

Estas posibles implicancias diagnósticas y pronósticas, son razones suficientes para sugerir que en pacientes con rinitis, sin síntomas de asma, se realice una exhaustiva evaluación clínica y funcional respiratoria por espirometría tal como lo propone la guía ARIA ${ }^{1}$. Nuestros resultados respaldarían la indicación de pruebas de función pulmonar en la forma persistente moderada-grave de rinitis, haciendo innecesaria su determinación rutinaria en otros grados de menor intensidad clínica. Estudios futuros con un mayor número de pacientes y reproducibles en otros centros nos permitirán confirmar esa propuesta.

El presente trabajo tiene la fortaleza de confirmar la afectación asintomática de la función pulmonar en niños y adolescentes con rinitis observada por otros autores, proporcional al grado clínico establecido por ARIA original y que el análisis se realizó ajustando un modelo logístico de otras co-variables potencialmente confusoras. Reconocemos las limitaciones que ofrece en la práctica clasificar los grados clínicos de rinitis. Como la investigación fue realizada en un centro de referencia, incluimos un número menor de pacientes con rinitis intermitente, que por sus características es menos frecuente como motivo de consulta.

Queda por establecer, con mayor número de estudios, las implicancias clínicas y pronósticas definitivas de nuestros hallazgos y si la intervención terapéutica sobre la rinitis puede contribuir a normalizar la función pulmonar de los casos afectados. 


\section{Conclusiones}

Existen alteraciones funcionales respiratorias en un importante porcentaje de niños con rinitis alérgica y no alérgica, sin asma, afectación que es más prevalente en la rinitis persistente moderadagrave. La presencia de algún parámetro de la función pulmonar alterado fue independiente de la condición de atopia y dependió tanto de la frecuencia como de la gravedad de los síntomas de rinitis. El defecto funcional es potencialmente reversible al broncodilatador en pacientes con rinitis moderada-grave.

\section{Contribución de los autores}

-Ricardo J. Saranz: Concepción y diseño de la idea, búsqueda, análisis e interpretación de la bibliografía. Redacción del artículo y revisión crítica y aprobación de la versión final.

-María F. Agresta: Concepción y diseño de la idea, búsqueda, análisis e interpretación de la bibliografía. Redacción del artículo y revisión crítica y aprobación de la versión final.

-Natalia A. Lozano: Concepción y diseño de la idea, búsqueda, análisis e interpretación de la bibliografía. Redacción del artículo y revisión crítica y aprobación de la versión final.

-Graciela Alegre: Realización de estudios complementarios. Búsqueda, análisis e interpretación de la bibliografía. Revisión crítica y aprobación de la versión final.

-Laura V. Sasia: Búsqueda, análisis e interpretación de la bibliografía. Revisión crítica y aprobación de la versión final.

-Luciano laniero: Revisión crítica y aprobación de la versión final.

-Yanina V. Berardi: Realización de estudios complementarios. Revisión crítica y aprobación de la versión final.

-Alejandro Lozano: Concepción y diseño de la idea, búsqueda, análisis e interpretación de la bibliografía. Redacción del artículo y revisión crítica y aprobación de la versión final.

\section{Limitaciones de responsabilidad:}

Los autores declaran que lo expresado en el manuscrito son de su responsabilidad y no de la Institución a la que representan.

\section{Fuentes de apoyo- Financiación:}

Los autores declaran que este trabajo científico se realizó con financiación de la Secretaría de Investigación de la Universidad Católica de Córdoba.

Conflicto de intereses:

Ninguno para declarar.

\section{Aspectos éticos:}

Expresados en el apartado "aspectos éticos" del manuscrito.

\section{Bibliografía}

1.Bousquet J, Khaltaev N, Cruz AA, Denburg J, Fokkens WJ, Togias A, et al. Allergic Rhinitis and its Impact on Asthma (ARIA) 2008 update (in collaboration with the World Health Organization, GA(2)LEN and AllerGen). Allergy 2008;63:8-160.

2. Ait-Khaled N, Pearce N, Anderson HR, Ellwood P, Montefort S, Shah J, and the ISAAC Phase Three Study Group. Global map of the prevalence of symptoms of rhinoconjunctivitis in children: The International Study of Asthma and Allergies in Childhood (ISAAC) Phase Three. Allergy 2009: 64:123-48.

3. Shaaban R, Zureik M, Soussan D, Neukirch C, Heinrich J, Sunyer $J$, et al. Rhinitis and onset of asthma: a longitudinal populationbased study. Lancet 2008;372:1049-57.

4.Braunstahl GJ. United airways concept: what does it teach us about systemic inflammation in airways disease? Proc Am Thorac Soc 2009; 6:652-4.

5.Boulay MV, Morin A, Laprise C, Boulet LP. Asthma and rhinitis: what is the relationship? Curr Opin Allergy Clin Immunol 2012, 12:449-54.
6. Kariyawasam HH, Rotiroti G. Allergic rhinitis, chronic rhinosinusitis and asthma: unravelling a complex relationship. Curr Opin Otolaryngol Head Neck Surg 2013, 21:79-86.

7.Agresta MF, Saranz RJ, Lozano A, Lozano NA. The relationship between rhinitis and asthma: is that all? Rev Fac Cien Med Univ Nac Cordoba 2014; 71:111-21.

8.Bertelsen RJ, Lødrup Carlsen KC, Carlsen K-H. Rhinitis in children:Co-morbidities and phenotypes. Pediatr Allergy Immunol 2010: 21: 612-22.

9. Chawes BL,, MD, Bønnelykke K, Kreiner-Møller Bisgaard B. Children with allergic and nonallergic rhinitis have a similar risk of asthma. J Allergy Clin Immunol 2010;126:567-73.

10. Thomas M, Kocevar VS, Zhang Q, Yin DD, Price D. Asthmarelated health care resource use among asthmatic children with and without concomitant allergic rhinitis. Pediatrics 2005;115;12934.

11. de Groot EP,Nijkamp A, Duiverman EJ, Brand PLP. Allergic rhinitis is associated with poor asthma control in children with asthma. Thorax 2012;67:582-7.

12. Deliu M, Belgrave D, Simpson A, Murray CS, Kerry G, Custovic $A$. Impact of rhinitis on asthma severity in school-age children. Allergy 2014; 69: 1515-21.

13. Padilla J,Uceda M, Ziegler O, Lindo F, Herrera-Pérez E, Huicho L. Association between Allergic Rhinitis and Asthma Control in Peruvian School Children: A Cross-Sectional Study. BioMed Res Int 2013; 1-7. http://dx.doi.org/10.1155/2013/861213.

14. Chawes BL. Upper and lower airway pathology in young children with allergic and non-allergic rhinitis. Dan Med Bull 2011; 58(5):1-23.

15. Ciprandi G, Cirillo I, Pistorio A. Impact of allergic rhinitis on asthma: effects on spirometric parameters. Allergy 2008;63:25560.

16. Ciprandi G, Capasso M. Association of childhood perennial allergic rhinitis with subclinical airflow limitation. Clin Exp Allergy 2010;40:398-402.

17. Ianiero L, Saranz RJ, Lozano NA, Lozano A, Sasia LV, Ramírez $M$, Cuestas $E$. Análisis de la curva flujo-volumen en niños y adolescentes con rinitis alérgica sin asma. Arch Argent Pediatr 2013;111(4):322-27

18. Capasso M, Vardicchio A, Ciprandi G. Impact of allergic rhinitis on asthma in children: effects on bronchodilation test. Allergy 2010;65:264-68.

19. Saranz RJ, Lozano A, Valero A,Lozano NA, Bovina Martijena MP, Agresta MF, laniero L, Ponzio MF. Impact of rhinitis on lung function in children and adolescents without asthma. Allergol Immunopathol (Madr) 2016;44:556-62.

20. Montoro J, Del Cuvillo A, Mullol J, Molina X, Dávila I, Ferrer M, et al. Validation of the modified allergic rhinitis and its impacton asthma (ARIA) severity classification in allergic rhinitis chil-dren: the PEDRIAL study. Allergy 2012;67:1437-42.

21. Eigenmann PA, Atanaskovic-Markovic M, O'B Hourihane J, Lack G, Lau S, Matricardi PM, et al. Testing children for allergies: why, how, who and when. Pediatr Allergy Immunol 2013: 24: 195209.

22. Miller MR, Hankinson J, Brusasco V, Burgos F, Casaburi R, Coates A, et al. Standardisation of spirometry. Eur Respir J 2005; 26:319-38.

23. Knudson RJ, Slatin RC, Lebowitz MD, Burrows B. The maximal expiratory flow-volume curve. Normal standards, variability, and effects of age. Am Rev Respir Dis 1976;113: 587-600.

24. Di Rienzo J.A., Casanoves F., Balzarini M.G., Gonzalez L., Tablada M., Robledo C.W. InfoStat versión 2013. Grupo InfoStat, FCA, Universidad Nacional de Córdoba, Argentina. URL http://www.infostat.com.ar

25. Lohia S, Schlosser RJ, Soler ZM. Impact of intranasal corticosteroids on asthma outcomes in allergic rhinitis: a metaanalysis. Allergy 2013;68:569-579.

26. Saranz RJ, Lozano A, Lozano NA, Sosa Aguirre AG, Alegre G. Mecanismos de la conexión nariz-pulmón. Methodo 2017;2 :3-15.

27. Ciprandi G ,Quaglini S, Giunta V ,Cirillo I. Assessment of the bronchodilation test by visual analog scale in the selection of patients with rhinitis for screening spirometry. J Investig Allergol Clin Immunol 2010; 20: 419-24. 
28. Skylogianni E, Triga M, Douros K, Bolis K, Priftis KN, Fouzas $S$,et al. Small-airway dysfunction precedes the development of asthma in children with allergic rhinitis. Allergol Immunpathol 2018;46:313-21.

29. Bouzigon E, Forabosco P, Koppelman GH, Cookson WO, Dizier $\mathrm{MH}$, Duffy $\mathrm{DL}$, et al. Meta-analysis of 20 genome wide linkage studies evidenced new regions linked to asthma and atopy. Eur $J$ Hum Genet 2010; 18:700-6.

30. Saranz RJ, Lozano A, Lozano NA, Ponzio MF, laniero L, Agresta MF. Pronóstico de las alteraciones pulmonares subclínicas de la rinitis. Alergia e Inmunol Clin 2016;35: 4-12. 